\title{
Dispensed Treatment Label Identifier Reference
}

National Cancer Institute

\section{Source}

National Cancer Institute. Dispensed Treatment Label Identifier Reference. NCI Thesaurus. Code C87823.

A character or string used to name or characterize the reference identifier for a dispensed treatment label. 